\title{
Hybrid exceptional point created from type-III Dirac point
}

\author{
L. Jin $\odot,,^{1, *}$ H. C. Wu, ${ }^{1}$ Bo-Bo Wei, ${ }^{2,3, \dagger}$ and Z. Song ${ }^{1}{ }^{1}$ \\ ${ }^{1}$ School of Physics, Nankai University, Tianjin 300071, China \\ ${ }^{2}$ School of Science and Engineering, The Chinese University of Hong Kong, Shenzhen, Shenzhen 518172, China \\ ${ }^{3}$ Center for Quantum Computing, Peng Cheng Laboratory, Shenzhen 518055, China
}

(Received 18 August 2019; revised manuscript received 12 January 2020; published 27 January 2020)

\begin{abstract}
Degeneracy points and exceptional points embedded in the energy band are distinct because of their topological features. We report a hybrid exceptional point formed through merging two ordinary exceptional points with opposite chiralities that were created from the type-III Dirac points emerging from a flat band. The hybrid exceptional point is induced by the destructive interference at the proper match between the non-Hermiticity and the synthetic magnetic flux. The degeneracy points and different types of exceptional points are distinguishable by their topological features of the global geometric phase accompanied by the scaling exponent of phase rigidity. Our findings not only pave the way for creating, moving, and merging exceptional points but also shed light on future investigations of non-Hermitian topological phases.
\end{abstract}

DOI: 10.1103/PhysRevB.101.045130

\section{INTRODUCTION}

Exceptional points (EPs) are non-Hermitian degeneracies [1-4], at which the system Hamiltonian is defective and eigenstates coalesce [5]. A parity-time $(\mathcal{P} \mathcal{T})$ symmetric phase transition and many intriguing dynamical phenomena occur at the EPs [6-20]. The properties of a non-Hermitian system dramatically change in the vicinity of EPs and are valuable for optical sensing [21-25]. Frequency sensing is enhanced because the responses of energy levels to the detuning perturbation are the square root near a two-state coalescence (EP2) and the cubic root near a three-state coalescence (EP3) in non-Hermitian systems, which is more efficient than the linear response near a diabolic point (DP) in Hermitian systems $[24,25]$.

The EPs possess distinct topology from DPs [26]. In a twolevel non-Hermitian system, the energy levels interchange after encircling the EP for one circle in the parameter space. The interchanged energy levels restore their original values after two circles of encircling and accumulate a geometric phase $\pm \pi$; the sign of the geometric phase depends on the circling direction, and the chirality of the EP is defined by the accumulated geometric phase under the counterclockwise encircling [27-36]. Dynamical encircling of EPs realizes a state switch and nonreciprocal topological energy transfer [37-40]; the dynamics depends on the starting and end points [41] and the homotopy of the encircling loop [42].

\footnotetext{
*jinliang@nankai.edu.cn

†weibobo@cuhk.edu.cn
}

Published by the American Physical Society under the terms of the Creative Commons Attribution 4.0 International license. Further distribution of this work must maintain attribution to the author(s) and the published article's title, journal citation, and DOI.
The EPs are different in their ways of coalescence as well as their topological properties [43]. In one aspect, the EPs are distinct in their orders: If more than two energy levels coalesce at the EP, the EP is called a high-order EP [44,45], where the excitation intensity presents the polynomial increase [46]. In another aspect, even the EPs with identical orders may dramatically differ from each other in the topological aspect. For example, when encircling a high-order EP with three-state coalescence (EP3) in the energy band of a square-root-type Riemann surface in the parameter space, two energy levels flip after encircling one circle; if an EP3 is in a cubic-roottype Riemann surface, when it is encircled, three circles are needed to restore the energy levels to their original values. The geometric phases associated with the two EP3s are different, which reflects the different topological features of two such types of EP3s [47]. An interesting question naturally arises: Do EP2s have different topologies? If so, how does one characterize their topological properties and distinguish them?

The manipulation of Dirac points in condensed-matter physics is an interesting and challenging task. The merging of Dirac points induces a topological phase transition and generates new types of Hermitian degeneracy; for example, two Dirac points with opposite topological charges can merge into a semi-Dirac point with linear and quadratic dispersions along two orthogonal directions [48-51]. In parallel, the merging of EPs leads to multifarious Hermitian and non-Hermitian degeneracies. It has been demonstrated that the merging of EPs may (i) lead to the DP [51], (ii) create the high-order EP [35,36], and (iii) form the hybrid EP [52,53]. The hybrid EP has linear and square-root dispersions along two orthogonal directions and carries integer topological charge, in contrast to the ordinary EP2 that carries half-integer topological charge [54].

The hybrid EP can be formed by merging either two ordinary EP2s with identical chirality (Fig. 1, plots I and II) or two ordinary EP2s with opposite chiralities (Fig. 1, plots III and IV). In this paper, we propose and investigate the latter 


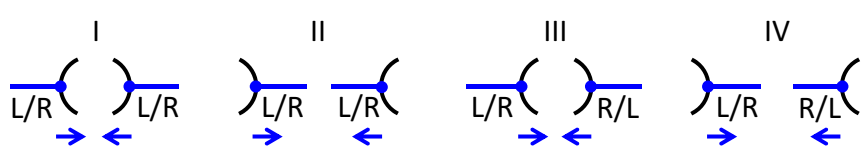

FIG. 1. Schematic of four types of EP2 merging, indicated by the blue arrows. The blue dots indicate the left- and right-chiral EP2s embedded in the branched energy bands; the blue lines indicate the Fermi arcs. The chirality refers to the geometric phase associated with the EP2 when it is encircled in the parameter space. The vertical axis indicates the real part of the energy levels, and the horizontal axis indicates a system parameter.

unexplored case by exploiting a non-Hermitian three-band lattice. The hybrid EP is created from type-III Dirac points emerging from a flat band of a three-band system enclosed in synthetic magnetic flux under the appropriate gain and loss. The EPs can merge into different types of EPs for different system parameters. Topological characterization employing the Berry phase and the non-Hermitian charge and vortex associated with the complex Riemann surface band structure of non-Hermitian systems [52,54] cannot fully distinguish all types of EPs; we employ the Berry phase and the phase rigidity scaling exponent to overcome this difficulty. Our findings are elaborated in a three-band system. The creating, moving, and merging of band touching points are investigated. The DPs and various types of EPs possess distinct topological properties; they are well distinguished from each other under the developed topological characterization (see Table I) and indicate different topological phases of the system. The topological features of EP mergers are unveiled.

\section{THE BAND STRUCTURE AND PHASE DIAGRAM OF THE THREE-BAND SYSTEM}

We consider a non-Hermitian three-band system with various configurations of EPs in the gapless phase [51]. The Hamiltonian reads

$$
H=\left(\begin{array}{ccc}
h_{z}+i \gamma & h_{x} & J e^{i \Phi} \\
h_{x} & 0 & h_{y} \\
J e^{-i \Phi} & h_{y} & -h_{z}-i \gamma
\end{array}\right) .
$$

The investigation of $H$ in the parameter space $\left(h_{x}, h_{y}, h_{z}\right)$ helps us grasp its topological properties. Since the couplings $h_{x}$ and $h_{y}$ play the same role, we take $h_{x}=h_{y}$ without loss of generality. To be concrete, Fig. 2(a) schematically illustrates a three-band lattice. Applying the Fourier transformation, the Bloch Hamiltonian of the three-band lattice shown in Fig. 2(a) is obtained in the form of $H$ with $h_{x}=h_{y}=v+R \cos k, h_{z}=$ $R \sin k$, where $k$ is the momentum (see Appendix A for more details). The trajectory of $\left(h_{x}, h_{z}\right)$ forms a closed circle in the

TABLE I. Topological characterization of the DP and EPs (see Appendixes B and C for more details).

\begin{tabular}{lccccc}
\hline \hline & DP & EP2(T) & EP2(I) & EP2 & EP3 \\
\hline $\pm w$ & 0 & 0 & 0 & $1 / 2$ & 1 \\
$v$ & 2 & 1 & $1 / 2$ & $1 / 2$ & 1 \\
\hline \hline
\end{tabular}
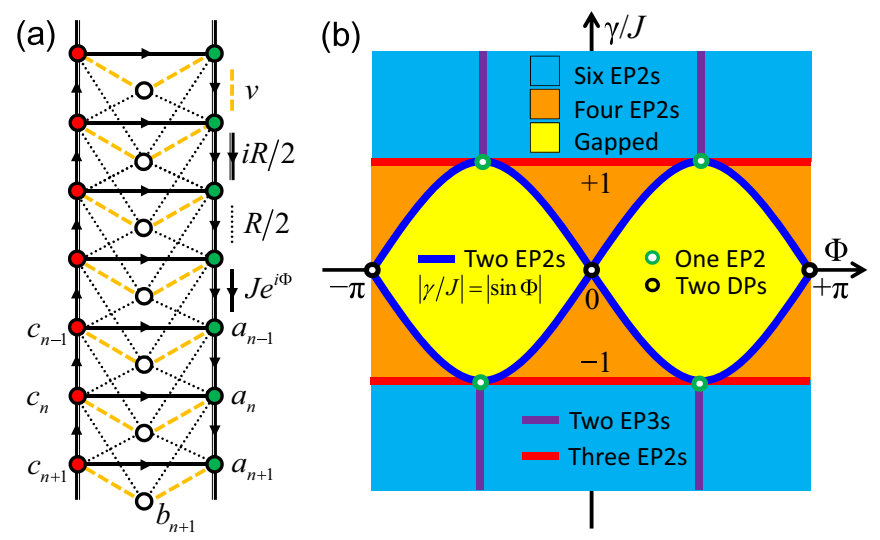

FIG. 2. (a) Horizontally $\mathcal{P} \mathcal{T}$-symmetric quasi-one-dimensional triangular lattice. $v, R, J$ are the coupling strengths among sublattices $a, b$, and $c . \Phi$ is the Peierls phase in the nonreciprocal coupling $J e^{ \pm i \Phi}$ between sublattices $a$ and $c$; the arrows indicate the phase direction of the couplings. The green (red) site indicates the gain (loss). (b) Phase diagram of $H$ in Eq. (1). The EP merging of Fig. 1, plot III, occurs when the system parameters are chosen at the red lines, and the merging of Fig. 1, plot IV, occurs when the system parameters are chosen at the blue lines.

$h_{x}-h_{z}$ parameter space; the topological features of the Bloch bands directly relate to the topological properties of the band touching points enclosed in the trajectory of $\left(h_{x}, h_{z}\right)$ [34]. Thus, the topological properties of the three-band lattice can be obtained by studying the band structure and topology of $H$ in Eq. (1).

In Fig. 2(a), the sublattices $a$ and $c$ are indirectly coupled through sublattice $b$ and are directly coupled through a nonreciprocal coupling $J e^{i \Phi}[36,55]$. The Peierls phase factor $e^{ \pm i \Phi}$ can be realized in various manners [56-64], which induces effective magnetic fluxes in the triangles but not the square plaquettes of the lattice. The synthetic magnetic fields have been experimentally realized in coupled optical resonators $[65,66]$. Sublattice $a(c)$ has gain (loss), and sublattice $b$ is passive. $\mathcal{P} \mathcal{T}$-symmetric systems can be investigated by employing passive system with different losses; sticking absorption material or cutting waveguide induces additional loss [67-69]. The flat band in a non-Hermitian lattice without synthetic magnetic flux was previously proposed through engineering the gain and loss [70]; in addition, it was demonstrated that proposing a spectrum entirely constituted by flat bands is possible with non-Hermitian couplings [71]. Alternatively, the flat band in the Hermitian systems remains in the nonHermitian situation with the proper match of synthetic magnetic flux and non-Hermiticity [72]. The synthetic magnetic flux provides a useful resource to generate different types of EPs and motivates us to study the problem of creating, moving, and merging EPs, in particular, the topological properties of different types of EPs.

We first consider $\gamma=0$; the lower band gap closes, and the lower two bands touch at a pair of DPs for $\Phi=0, \pi$ [73] and $h_{z}=0$. The DPs are two Dirac points at the peaks of two type-III Dirac cones, which are the critically titled type-I Dirac cones with a flat-band line Fermi surface. The type-III Dirac cone associated with a line Fermi surface differs from 


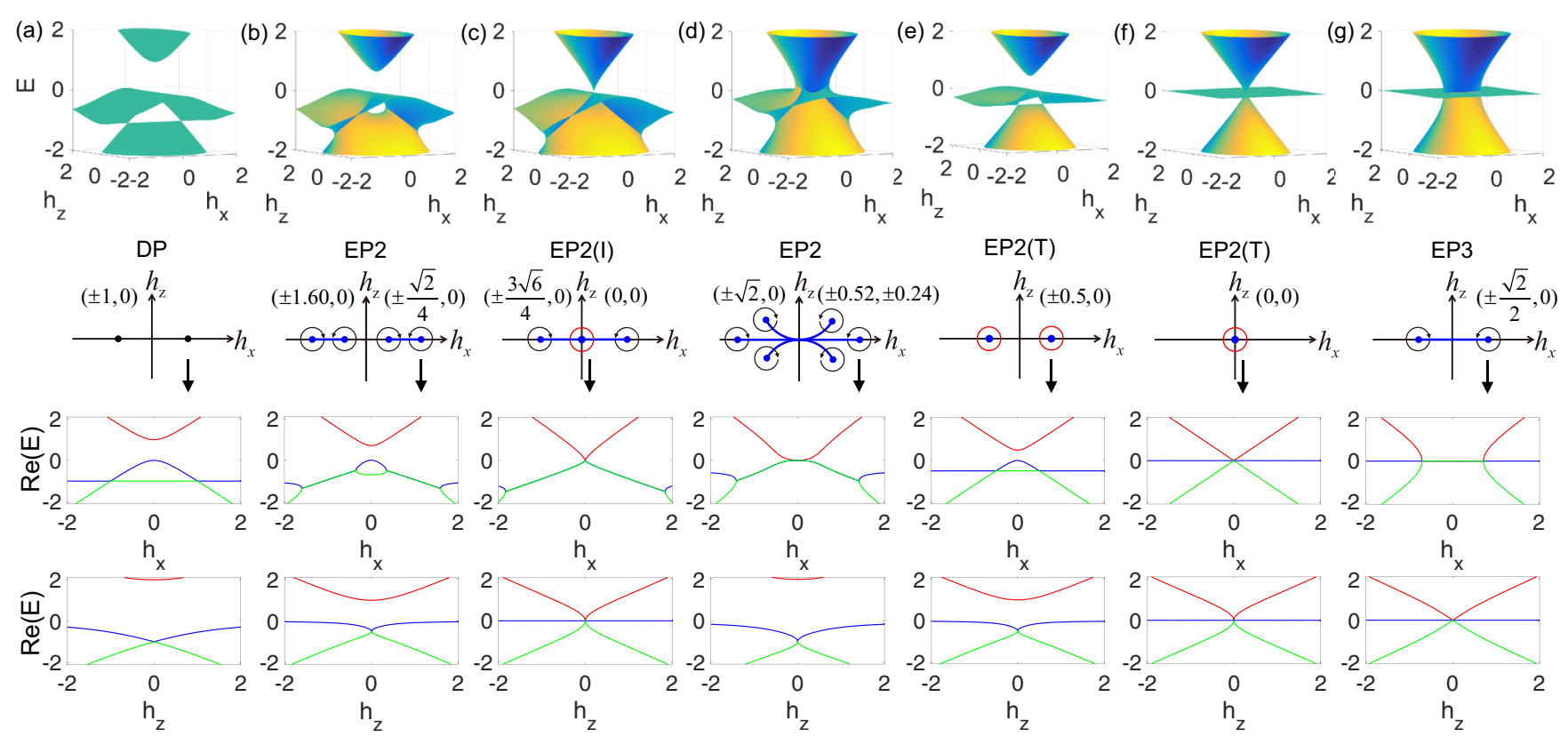

FIG. 3. Energy bands and EP distribution in the parameter space for the seven typical gapless phases in the phase diagram in Fig. 2(b). In the top panel, two axes in the horizontal plane represent $h_{x}$ and $h_{z}$, while the vertical axis represents the real part of the eigenvalues; the color indicates the imaginary part of the eigenvalues. (a) $\Phi=0, \gamma=0$ [black circle in Fig. 2(b)], (b) $\Phi=0, \gamma=\sqrt{2} / 2$ [orange region in Fig. 2(b)], (c) $\Phi=0, \gamma=1$ [red line in Fig. 2(b)], (d) $\Phi=\pi / 3, \gamma=\sqrt{2}$ [cyan region in Fig. 2(b)], (e) $\Phi=\pi / 3, \gamma=\sqrt{3} / 2$ [blue line in Fig. 2(b)], (f) $\Phi=\pi / 2, \gamma=1$ [green circle in Fig. 2(b)], and (g) $\Phi=\pi / 2, \gamma=\sqrt{2}$ [purple line in Fig. 2(b)]. The coupling $J=1$ in all plots. Types of EPs (blue dots) are illustrated in the middle panel. The EPs at $\left(h_{x}, h_{z}\right)$ are marked. The $+1(-1)$ chirality of EPs is indicated by the counterclockwise (clockwise) arrow; the solid blue lines are Fermi arcs connected to the EPs. The real spectra along both the $h_{x}$ and $h_{z}$ directions for the corresponding EPs are depicted in the bottom panels.

the type-I Dirac cone associated with a point Fermi surface as well as the type-II Dirac cone associated with a Fermi surface with two crossed lines [74-76]. The band touching creates a flat band $-e^{i \Phi} J$ due to the destructive interference at sublattice $b$. The two lower bands have isotropic linear dispersion near the Dirac points, as shown in the bottom panel of Fig. 3(a).

The DPs disappear when gain and loss are introduced $(\gamma \neq 0)$, and the band touching points become EPs. $H$ can exhibit rich band structures featured by different types of EPs. Figure 2(b) depicts the phase diagram; it shows the number of DPs (EPs) in the parameter plane $h_{x}-h_{z}$ at certain fixed $\Phi$ and $\gamma / J$. The types of EPs vary in different regions of $\gamma-\Phi$. The change in types of EPs indicates the topological phase transition, and the different configurations of multiple EPs represent different topological phases of $H$ [51]. Typical energy bands in the $h_{x}-h_{z}$ plane are exemplified in Fig. 3.

By introducing gain and loss $(\gamma \neq 0)$, the two Dirac points [Fig. 3(a)] can split into two pairs of ordinary EP2s with opposite chiralities [Fig. 3(b)]. Hybrid EP2 is formed by merging a pair of ordinary EP2s with opposite chiralities at an appropriate match between non-Hermiticity and synthetic magnetic flux [Figs. 3(e) and 3(f)]. The distribution of different types of EPs is indicated in the middle panel. At the band touching points, the system parameters satisfy

$$
\begin{gathered}
{\left[3 p^{2}-\left(2 \gamma h_{z}\right)^{2}\right] \gamma h_{z}=0,} \\
4 p^{3}-27 q^{2}-12 p\left(2 \gamma h_{z}\right)^{2}=0,
\end{gathered}
$$

where $p=2 h_{x}^{2}+J^{2}-\gamma^{2}+h_{z}^{2}$ and $q=2 h_{x}^{2} J \cos \Phi$. For $h_{x} \neq$ $h_{y}, h_{x}^{2}$ is replaced by $h_{x} h_{y}$ in $p, q$.

Three types of EP2s exist in the three-band system: (i) One is the ordinary EP2, which is the singularity point in the Riemann surface of the square-root type and has chirality. (ii) The second is EP2(T), which is a merger of two ordinary EP2s with opposite chiralities. Two relevant bands touch at EP2(T), and their real parts are gapped in the vicinity of EP2(T); the merging schematic is indicated in Fig. 1, plot IV. (iii) The last is EP2(I), which is also constituted by merging two ordinary EP2s with opposite chiralities. Two relevant bands intersect; the merging schematic is indicated in Fig. 1, plot III. EP2(T) has anisotropic dispersions, being linear (square root) along $h_{x}$ $\left(h_{z}\right)$, while EP2(I) has isotropic square-root dispersions along both $h_{x}$ and $h_{z}$. If $H$ has chiral symmetry, the EPs of $H$ become EP3s. Figure 3 depicts the energy bands and schematically illustrates all seven typical EP configurations in the gapless phase. The central two ordinary EP2s in Fig. 3(b) merging into one EP2(I) in Fig. 3(c) is merging of the type in Fig. 1, plot III, and the four ordinary EP2s in Fig. 3(b) merging into two hybrid EP2(T)s in Fig. 3(e) is merging of the type in Fig. 1, plot IV.

The EPs may disappear when the bands are gapped for $\Phi \neq 0, \pm \pi$. This happens at weak non-Hermiticity $|\gamma / J|<$ $|\sin \Phi|$ [yellow region in Fig. 2(b)], where three bands are gapped. The gain and loss compress the band gaps; the lower two bands touch at the EPs when $|\gamma / J|=|\sin \Phi|$, and the appropriate non-Hermiticity awakens the destructive interference and reproduces the flat band. The flat-band energy is 
altered to $-J \cos \Phi[72]$, and the band touching points become two EP2(T)s rather than two DPs. As the gain and loss rates $\gamma$ increase, the band gaps vanish, and all three bands intersect when $|\gamma / J| \geqslant 1$.

\section{TOPOLOGICAL CHARACTERIZATION OF BAND TOUCHING POINTS}

The geometric (Berry) phase of the energy band is relevant to the topological features of DPs and EPs. The generalized geometric phase for the non-Hermitian systems is defined as $[77,78]$

$$
\Gamma_{n}=i \oint_{\mathrm{C}}\left\langle\phi_{n}(k)\left|\nabla_{k}\right| \psi_{n}(k)\right\rangle d k,
$$

where $n$ is the band index. $\left|\psi_{n}(k)\right\rangle$ and $\left|\phi_{n}(k)\right\rangle$ are the eigenstates of Hamiltonians $H$ and $H^{\dagger}$ and form a biorthonormal basis $\left\langle\phi_{n}(k) \mid \psi_{n^{\prime}}(k)\right\rangle=\delta_{n n^{\prime}}$. The integration is performed over a loop $C$ in the parameter space. For DPs and EP2s, which are relevant for only two bands, the irrelevant third band regains its original eigenvalue when the loop $C$ of system parameters encircles a band touching DP or EP for one circle; the corresponding eigenstate accumulates a zero geometric phase. When the bands are tangled in the presence of Hermitian or non-Hermitian band degeneracies [79,80], the non-Abelian Berry connections $A_{m n}=\left\langle\phi_{m}(k)\left|\nabla_{k}\right| \psi_{n}(k)\right\rangle$ characterize the topological properties of the energy bands [80-82]. The global geometric phase $\Theta=\sum_{n=1}^{3} \Gamma_{n}$ is a topological invariant $[80,83,84]$. The winding number $w=\Theta /(m \pi)$ characterizes the topology of band touching points, where $m$ is the number of relevant bands.

Moreover, the global geometric phase is unable to distinguish the topology of all different EPs and needs the assistance of phase rigidity,

$$
r=\left|\left\langle\psi_{n}^{*} \mid \psi_{n}\right\rangle /\left\langle\psi_{n} \mid \psi_{n}\right\rangle\right|
$$

The phase rigidity $r$ describes the mixing of different states [85]. In a Hermitian system with a real matrix, the phase rigidity is 1 . When extended to the non-Hermitian system, the defective eigenstate is self-orthogonal, and the phase rigidity at the EPs reduces to zero [86]. The phase rigidity has a scaling law in the vicinity of EPs, $\left|r_{\mathrm{EP}}-r\right| \propto\left(\gamma_{\mathrm{EP}}-\gamma\right)^{\nu}$. The phase rigidity scaling exponent $v$ characterizes the response manner of energy bands when approaching the EPs along the parameter $\gamma$, while the geometric phase we discuss characterizes the topological features of energy bands around EPs in the parameter space at fixed $\gamma$.

Table I summarizes the winding number and the phase rigidity scaling exponent along $\gamma$ for the DP and all of the types of EPs. We turn to discussing the details of the topological properties of the band touching points. The following discussions are organized in order of gain and loss increase. The creating, splitting, moving, and merging of band touching points and their topological features are presented. We focus on the topological features of two types of unexplored twostate coalescence: EP2(T) and EP2(I); the details of the topological features of other band touching points are provided in the Appendixes B and C.

Two DPs appear at $\left(h_{x}, h_{z}\right)=( \pm 1,0)$ for $\Phi=0$ at $\gamma=$ 0 ; as depicted in Fig. 3(a), they are type-III Dirac points
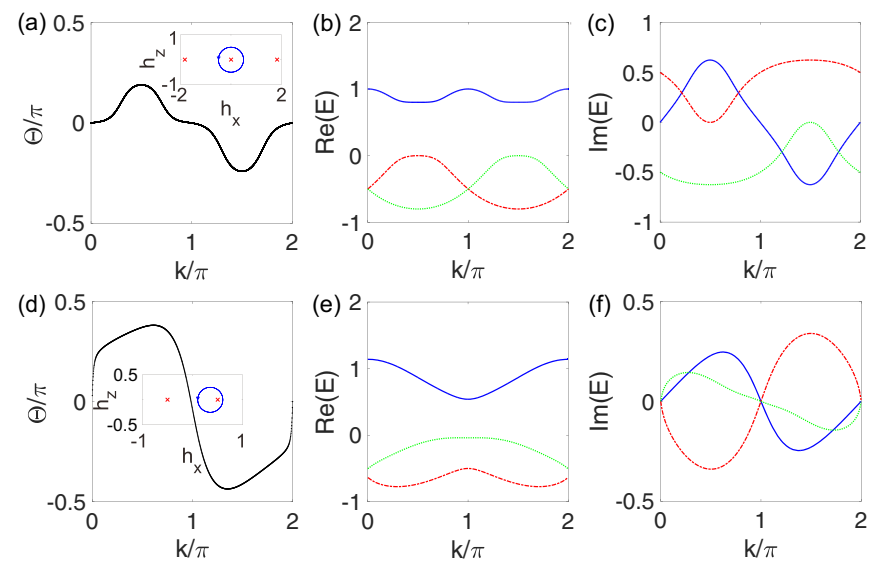

FIG. 4. (a)-(c) Encircling an EP2(I) at $J=1, \Phi=0$, $\gamma=1, v=0, R=1 / 2$ [Fig. 3(c)]. (d)-(f) Encircling an EP2(T) at $J=1, \Phi=\pi / 3, \gamma=\sqrt{3} / 2, v=\sqrt{2} / 4, R=1 / 4$ [Fig. 3(e)]. The EPs are marked by the red crosses, and the trajectory of the encircling in the parameter space is represented by the blue circle (insets); the loop radius is $R$, centered at $(v, 0)$.

embedded in the flat band and appear in the region marked by the black circles in the phase diagram in Fig. 2(b). The geometric phase for each of the two degenerate bands is zero when encircling either DP for one circle; the geometric phase for the irrelevant upper band is also zero. The phase rigidity scaling exponent is $v=2$ (see Fig. 8 in Appendix C).

The band gap between the lower two bands is closed in the presence of the flat band in Fig. 3(a). When the gain and loss are introduced $(\gamma \neq 0)$, the energy bands become even closer, and each DP splits into two ordinary EP2s with opposite chiralities at the weak non-Hermiticity $|\gamma / J|<1$. This describes the orange region in the phase diagram in Fig. 2(b). Figure 3(b) depicts the band spectrum in the situation $\gamma / J=$ $\sqrt{2} / 2<1$. The four ordinary EP 2 s are on the $h_{z}=0$ axis, and their chiralities are opposite with respect to $h_{x}=0$, as illustrated in the middle panel. The eigenvalues of two relevant coalescence states are $4 \pi$ periodic in $k$ when encircling the ordinary EP2s, but the period of the third state is $2 \pi$. After one circle of encircling, two relevant coalescence states exchange, and the third state regains its original eigenvalue; the global geometric phases $\Theta$ accumulated by the three bands equal $+\pi$ $(-\pi)$ for the ordinary EP2 of $+1(-1)$ chirality (see Fig. 6 in Appendix B), and two circles of encircling yield a $+\pi(-\pi)$ geometric phase for either relevant band. The phase rigidity scaling exponent associated with the ordinary EP2s is equal to $v=1 / 2$ (see Fig. 9 in Appendix C).

As the gain and loss rates $\gamma$ increase, the two central ordinary EP2s become closer, but the outer two ordinary EP2s become far away. At $|\gamma / J|=1$, the two central ordinary EP2s with opposite chiralities meet and merge into an EP2(I) at $\left(h_{x}, h_{z}\right)=(0,0)$ [52], and the system enters the region marked by the red lines in the phase diagram in Fig. 2(b) and has three EP2s [Fig. 3(c)] with an identical scaling exponent $v=1 / 2$, although they possess distinct topology [Figs. 5(a)-5(c)]. At the EP2(I), although three levels have identical zero energy, only two levels coalesce, and they degenerate with the third level; the system is defective, with one eigenstate missing. The energy levels regain their original values after encircling 

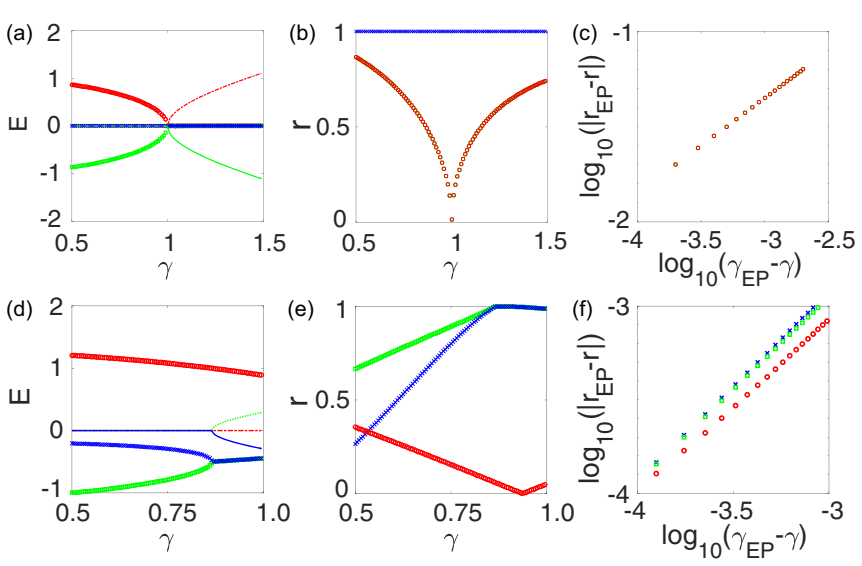

FIG. 5. Energy, phase rigidity, and scaling exponent (a)-(c) in the vicinity of an EP2(I) in Fig. 3(c) at $h_{x}=0, h_{z}=0, J=1, \Phi=$ 0 and (d)-(f) in the vicinity of an EP2(T) in Fig. 3(e) at $h_{x}=$ $1 / 2, h_{z}=0, J=1, \Phi=\pi / 3$. The real (imaginary) part is indicated by the symbols (lines). The scaling exponent is $v=0.5$ for an EP2(I) and is $v=1.0$ for an EP2(T).

the EP2(I) for one circle; states switch twice for the two lower levels; the geometric phases for the two lower levels are still opposite, being $\pi$ and $-\pi$, respectively, and the winding number for the global geometric phase is zero. The global geometric phase for encircling the EP2(I) at $\left(h_{x}, h_{z}\right)=(0,0)$ is depicted in Fig. 4(a). The real and imaginary parts of eigenenergies of $H_{k}$ for the encircling process are depicted in Figs. 4(b) and 4(c). The other two EP2s $( \pm 3 \sqrt{6} / 4,0)$ are ordinary EP2s. The EP2(I) in the center is connected to the other two ordinary EP2s by two Fermi arcs [87].

In the region $|\sin \Phi|<|\gamma / J|<1$, four ordinary EPs with two opposite chiralities in pairs locate on the two sides of $h_{z}=0$ [Fig. 3(b)]. By changing $\Phi$, each pair of EPs can merge into an EP2(T) when $|\gamma / J|=|\sin \Phi|$ for $0<|\Phi|<$ $\pi / 2$ [Fig. 3(e)]; this is indicated by the blue lines in the phase diagram in Fig. 2(b). Two valleys (peaks) appear in the middle (lower) band; the apexes of the valleys and peaks touch, two EP2(T)s are formed at the appropriate match between the effective magnetic flux $\Phi$ and non-Hermiticity $\gamma$, and the flat band reappears. In contrast to two DPs, the isolated band touching points are EP2(T)s located at $h_{x}^{2}=J^{2} \cos ^{2} \Phi$. In the parameter space, the global geometric phase for encircling the EP2(T) $\left(h_{x}, h_{z}\right)=(1 / 2,0)$ in the counterclockwise direction is depicted in Fig. 4(d). Figures 4(e) and 4(f) are the real and imaginary parts of eigenenergies of $H_{k}$. No state switch occurs when encircling the EP2(T) in the parameter space for one cycle, and the global geometric phase is zero; the EP2(T) has a winding number of zero without chirality, and the scaling exponent of the phase rigidity is $v=1$ [Figs. 5(d)-5(f)]. In the region of weak non-Hermiticity $|\gamma / J|<|\sin \Phi|$, the three bands are gapped without band touching.

For $|\gamma / J|>1$, the central EP2(I) vanishes and splits into four ordinary EP2s with two +1 and two -1 chiralities in the $h_{z} \neq 0$ region, and six ordinary EP2s exist, provided that $H$ is not chiral symmetric [Fig. 3(d)]; the phase is the cyan region in the phase diagram in Fig. 2(b). Among the six ordinary EP2s, three of them in the region $h_{x}>0\left(h_{x}<0\right)$ have +1 $(-1)$ chirality. The upper two bands coalesce at four ordinary
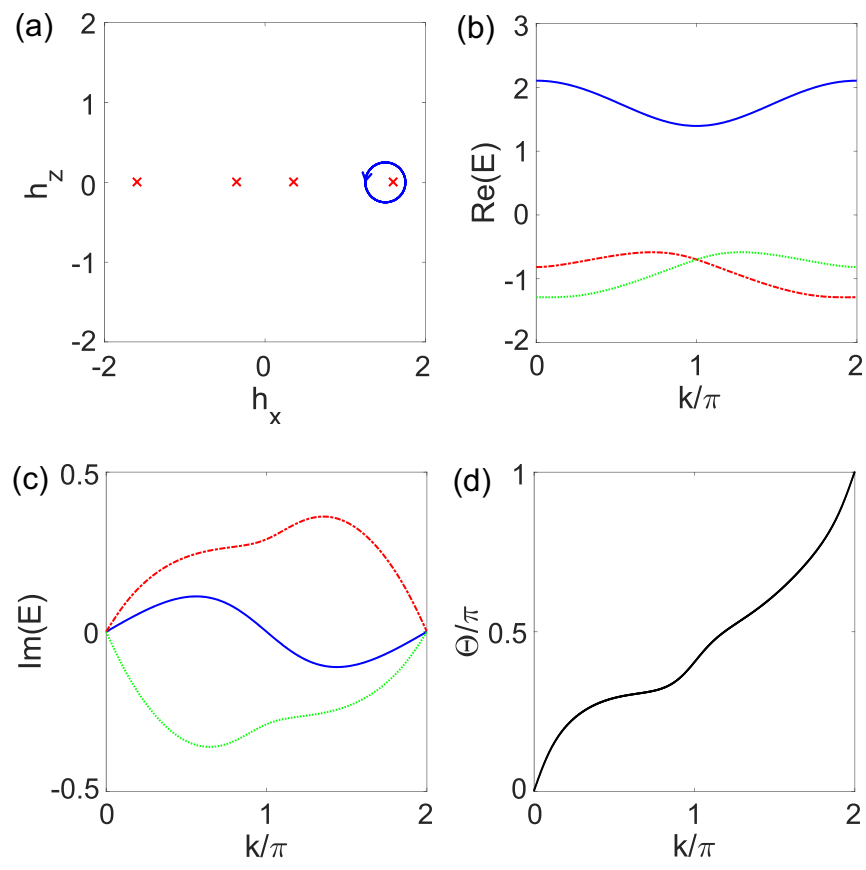

FIG. 6. Encircling an ordinary EP2 in Fig. 3(b) with a chirality of +1 . (a) The trajectory of the encircling in the parameter space is indicated by the blue circle; the EPs are marked by the red crosses. (b) and (c) The real and imaginary parts of energy and (d) the global geometric phase. The system parameters are $J=1, \Phi=0, \gamma=$ $\sqrt{2} / 2, v=3 / 2, R=1 / 4$.

EP2s $\left(h_{z} \neq 0\right)$, and the lower two bands coalesce at the other pair of ordinary EP2s on the $h_{z}=0$ axis. The energy bands with six ordinary EP2s are shown in Fig. $3(\mathrm{~d}) ; \Phi=\pi / 3$ is chosen in order to observe all the EP2s within the region $[-2,2]$ in the parameter space.

The three-band Hamiltonian $H$ is chiral symmetric when $J=0$ or $\Phi= \pm \pi / 2, \mathcal{C H C}^{-1}=-H$, where $\langle m|\mathcal{C}| n\rangle=$ $(-1)^{m+1} \delta_{m, 4-n}$. A zero-mode flat band is formed under the chiral symmetry. The upper and lower bands in Fig. 3(f) constitute a hybrid conical surface, the projection of which on the $E-h_{x}\left(E-h_{z}\right)$ plane is a cone of square-root repulsion that differs from a Dirac cone or a semi-Dirac cone [88-90]. At $|\gamma / J|<1$, the spectrum is gapped, and EP vanishes. At $|\gamma / J|=1$, two EP2(T)s merge to a single EP2(T) at $(0,0)$; the phase is represented by the green circles in the phase diagram in Fig. 2(b). At $|\gamma / J|>1$, the system has one pair of EP3s with opposite chiralities at $\left(h_{x}, h_{z}\right)=\left( \pm \sqrt{\left(\gamma^{2}-J^{2}\right) / 2}, 0\right)$ [Fig. 3(g)]; the phase is indicated by the purple lines in the phase diagram in Fig. 2(b). After encircling an EP3 for one circle, the upper and lower bands switch, and two circles are needed to restore the original eigenvalues. The global geometric phase accumulated is $+3 \pi(-3 \pi)$ after encircling an EP3 of chirality $+1(-1)$ for one circle (see Fig. 7 in Appendix B). The scaling exponent of the phase rigidity close to the EP3 is $v=1$ (see Fig. 10 in Appendix C).

\section{DISCUSSION AND CONCLUSION}

The topological characterization of band touching points applies for all types of EPs and is not limited to the DP, 

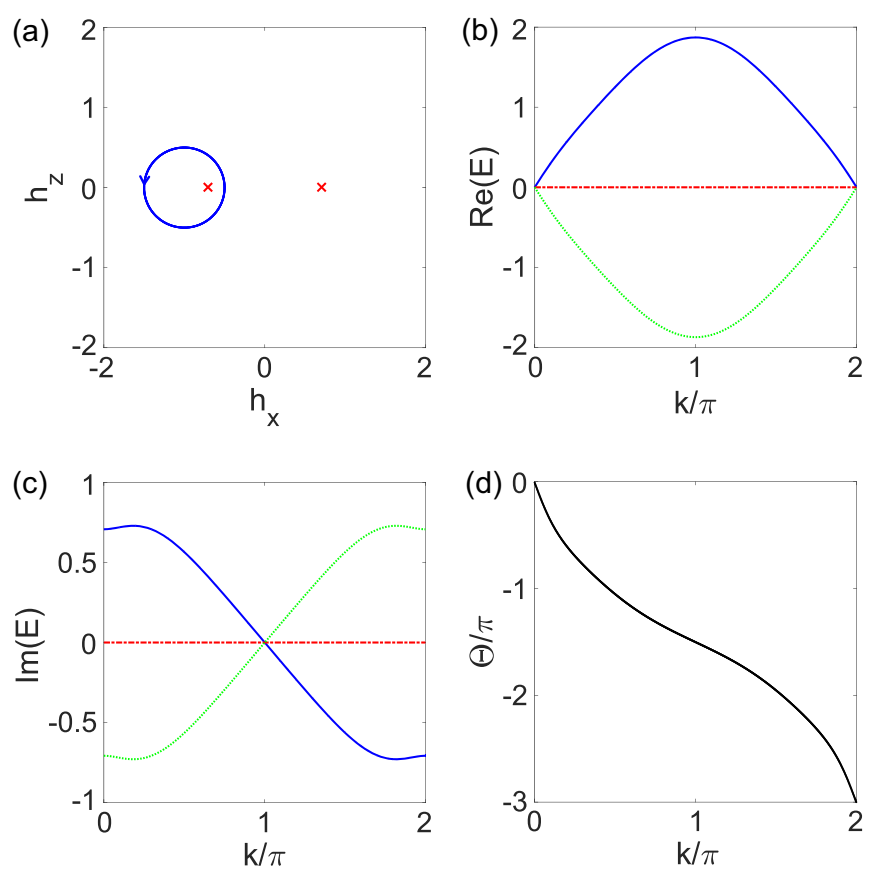

FIG. 7. Encircling an EP3 in Fig. 3(g) with a chirality of -1 . (a) The trajectory of the encircling in the parameter space is indicated by the blue circle; the EPs are marked by the red crosses. (b) and (c) The real and imaginary parts of energy and (d) the global geometric phase. The system parameters are $J=1, \Phi=\pi / 2, \gamma=$ $\sqrt{2}, v=-1, R=1 / 2$.

the ordinary EP2/EP3, and the hybrid EP2s presented in the lattice model of Fig. 2(a), as listed in Table I. For EP2 merging of the types in plots I and II in Fig. 1, the geometric phase is $\pi$, and the winding number is $|w|=1$. Different types of EPs are distinguishable by their topological features.

A hybrid EP generally presents in the non-Hermitian systems that possess a flat band with EPs embedded. The EP embedded in a flat band is a hybrid EP if two-dimensional parameter space is considered [71]. Notably, $H$ can describe the non-Hermitian Lieb lattice with additional gain and loss $\gamma$. The synthetic magnetic flux $J e^{i \Phi}$ can be induced by the spin-orbital coupling [64]. The Bloch Hamiltonian of the non-Hermitian Lieb lattice has the form of $h_{x}=$ $2 J_{x} \cos \left(k_{x} / 2\right), h_{y}=2 J_{y} \cos \left(k_{y} / 2\right), h_{z}=0$ [91]. In addition, a hybrid EP can appear in the absence of a flat band, and it is possible to create a hybrid EP of arbitrarily high order with asymmetric couplings $[53,92]$. The asymmetric coupling has a connection to the gain and loss in non-Hermitian systems. In practice, the gain and loss associated with the effective magnetic flux equivalently induce asymmetric coupling and nonreciprocity in the non-Hermitian systems $[55,93,94]$.

In conclusion, we proposed the hybrid EP2 by merging two ordinary EP2s with opposite chiralities, which are created from the type-III Dirac points emerging from a flat band through introducing proper gain and loss. The topology of the degenerate (exceptional) point is characterized by the winding number $w$ associated with the global geometric phase and phase rigidity scaling exponent $v$. The topological properties of different EP2 mergers were unveiled; the change in topological features associated with the merging of EPs indicates the topological phase transition. Our findings pave the way for creating, moving, and merging EPs and are valuable for future studies on the non-Hermitian topological phase of matter. In the future, further investigations on the dynamical encircling of hybrid EPs [53], the creating, moving, and merging of high-order EPs [36,51], and the topological edge states [95] would be of great interest.

\section{ACKNOWLEDGMENTS}

We acknowledge the support of the National Natural Science Foundation of China (Grants No. 11975128, No. 11605094, No. 11604220, and No. 11874225). B.-B.W. also acknowledges the President's Fund of the Chinese University of Hong Kong, Shenzhen.

\section{APPENDIX A: TRIANGULAR LATTICE}

In this Appendix, we show the real-space lattice Hamiltonian for the three-band Bloch Hamiltonian $H$ in Eq. (1). The three-band tight-binding lattice consists of three sublattices; sublattice $a(c)$ has gain (loss), and sublattice $b$ is passive. Sublattices $a$ and $c$ have nonreciprocal nearestneighbor couplings. The phase factors in the couplings are opposite, indicated by the arrows. Sublattices $a$ and $c$ are coupled indirectly through sublattice $b$ and directly through a nonreciprocal coupling $J e^{i \Phi}$, which can be realized in cold atomic gases by inducing the spin-orbital interaction and can be realized by optical path imbalance, dynamic modulation, and photon-phonon interaction in optics. The triangular lattice Hamiltonian $H_{T L}$ in the real space is given by

$$
\begin{aligned}
H_{T L}= & \sum_{j=1}^{N}\left(-\frac{i R}{2} a_{j}^{\dagger} a_{j+1}+\frac{i R}{2} c_{j}^{\dagger} c_{j+1}+\text { H.c. }\right) \\
& +\left(\frac{R}{2} a_{j}^{\dagger} b_{j-1}+\frac{R}{2} a_{j}^{\dagger} b_{j+1}+\text { H.c. }\right) \\
& +\left(\frac{R}{2} c_{j}^{\dagger} b_{j-1}+\frac{R}{2} c_{j}^{\dagger} b_{j+1}+\text { H.c. }\right) \\
& +\left(J e^{i \Phi} a_{j}^{\dagger} c_{j}+v a_{j}^{\dagger} b_{j}+v a_{j}^{\dagger} c_{j}+\text { H.c. }\right) \\
& +i \gamma a_{j}^{\dagger} a_{j}-i \gamma c_{j}^{\dagger} c_{j} .
\end{aligned}
$$

The couplings strengths are $v, R / 2$, and $J$. The nonreciprocal couplings $\pm i R / 2$ lead to an effective magnetic flux $\pi$ enclosed in each square plaquette. The gain and loss rates are $\gamma$. In experimental studies, it is not necessary to induce the gain to balance the loss in the investigations of $\mathcal{P} \mathcal{T}$-symmetric lattices; using the loss-only passive systems brings convenience [67-69]. For example, we can introduce $\{0,-i \gamma,-2 i \gamma\}$ instead of $\{i \gamma, 0,-i \gamma\}$ in sublattices $a, b$, and $c$. By offsetting an imaginary energy $+i \gamma$ to the on-site terms $\{0,-i \gamma,-2 i \gamma\}$, we obtain the $\mathcal{P} \mathcal{T}$-symmetric Hamiltonian.

Applying the Fourier transformation,

$$
a_{k}=\sum_{j=1}^{N} e^{i k j} a_{j}, \quad b_{k}=\sum_{j=1}^{N} e^{i k j} b_{j}, \quad c_{k}=\sum_{j=1}^{N} e^{i k j} c_{j},
$$

where the discrete momentum is $k=2 \pi n / N$ (integer $n \in$ $[1, N]) ; a_{j}, b_{j}$, and $c_{j}$ are the annihilation operators that 

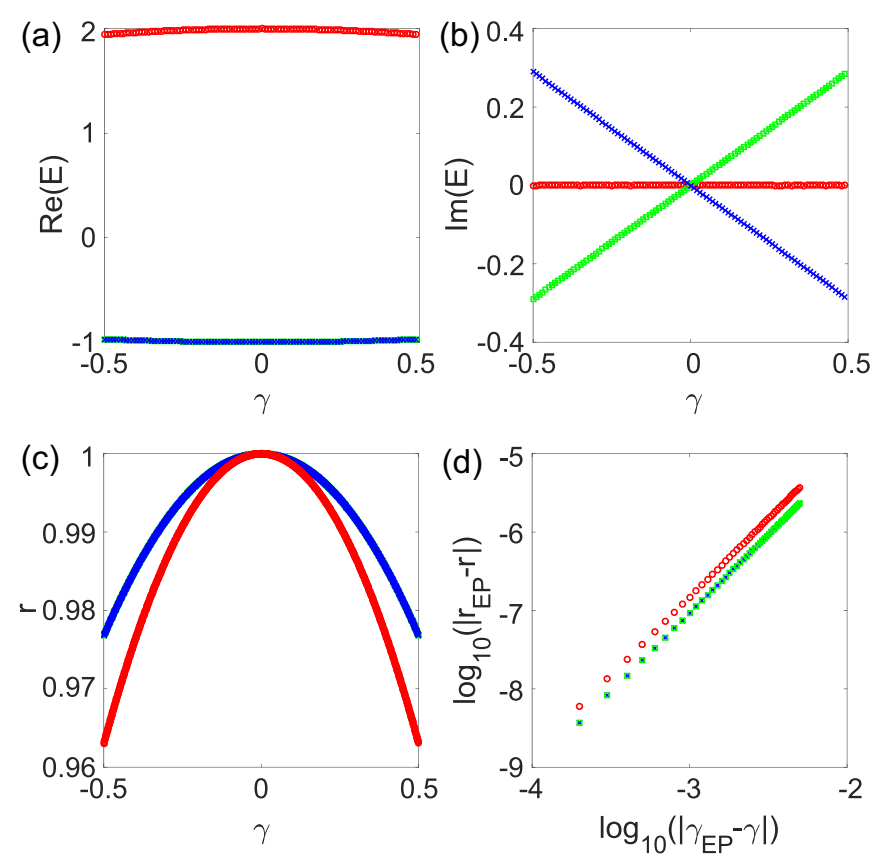

FIG. 8. (a) and (b) Spectrum, (c) phase rigidity, and (d) scaling law at a DP in Fig. 3(a). The system parameters are $h_{x}=1, h_{z}=$ $0, J=1, \Phi=0$. The phase rigidity scaling exponents for all the states are $v=2.0$.

satisfy the periodical boundary condition $a_{N+1}=a_{1}, b_{N+1}=$ $b_{1}$, and $c_{N+1}=c_{1}$. In the momentum space, the lattice Hamiltonian is expressed as $H_{T L}=\sum_{k} H_{k}$, with $h_{x}=v+$ $R \cos k$ and $h_{z}=R \sin k$. The Bloch Hamiltonian $H_{k}$ in the
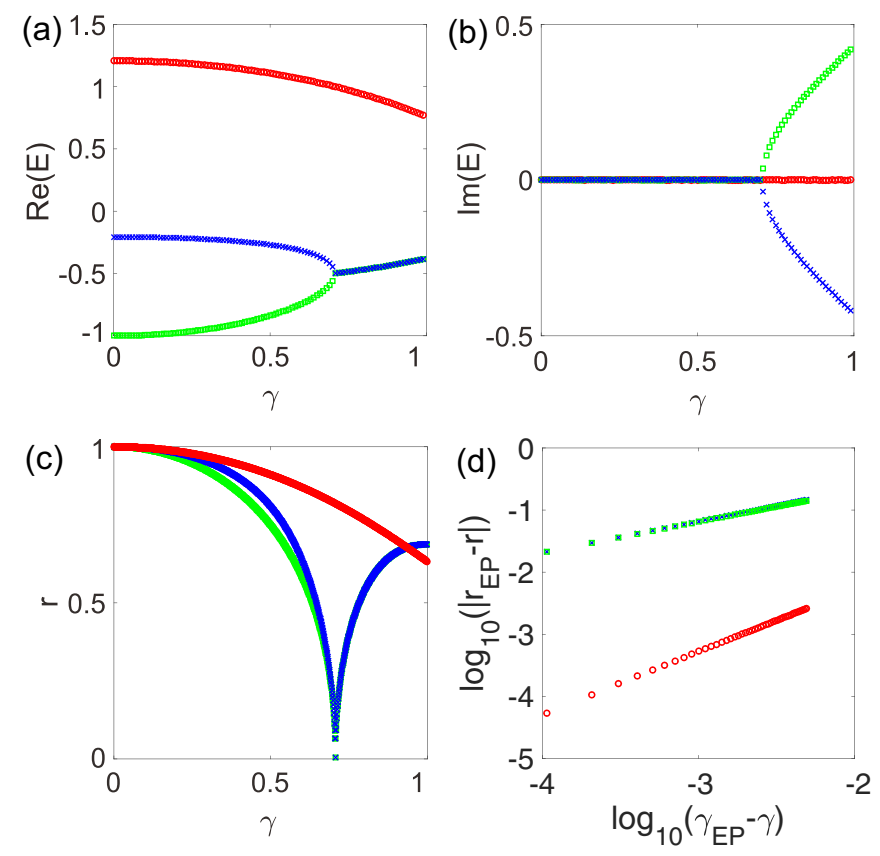

FIG. 9. (a) and (b) Spectrum, (c) phase rigidity, and (d) scaling law at an ordinary EP2 in Fig. 3(b). The system parameters are $h_{x}=\sqrt{2} / 4, h_{z}=0, J=1, \Phi=0$. The scaling exponents for the coalesced states (green squares and blue crosses) are $v=0.5$; the scaling exponent for the third state (red circles) is $v=1.0$.
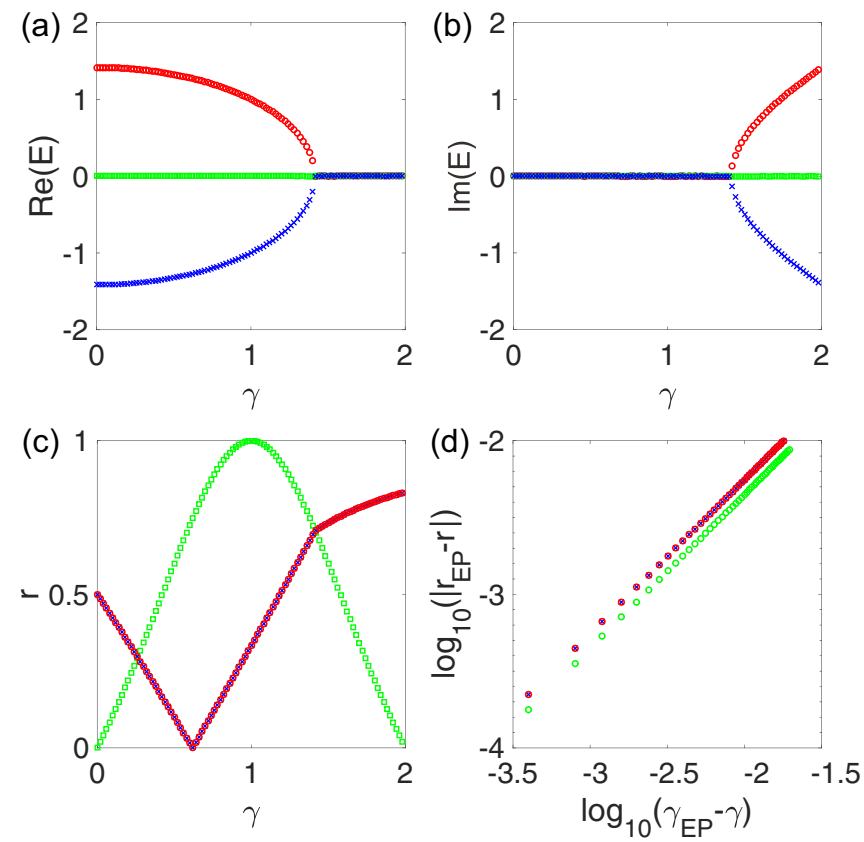

FIG. 10. (a) and (b) Spectrum, (c) phase rigidity, and (d) scaling law at an EP3 in Fig. 3(g). The system parameters are $h_{x}=\sqrt{2} / 2, h_{z}=0, J=1, \Phi=\pi / 2$. The scaling exponents for all states are $v=1.0$.

momentum space is a $3 \times 3$ non-Hermitian Hamiltonian in the form of Eq. (1) in the main text for $h_{x}=h_{y}$.

\section{APPENDIX B: GEOMETRIC PHASE}

In this Appendix, we show the geometric phase associated with the band touching points, including the ordinary EP2 and EP3.

The global geometric phase for a non-Hermitian system is $Q=i \sum_{n=1}^{3} \oint_{\mathrm{C}}\left\langle\phi_{n}(k) \mid d \psi_{n}(k)\right\rangle[83,84]$. The integration is performed over a loop $C$ in the parameter space. The trajectory of $\left(h_{x}, h_{z}\right)$ forms a closed circle $C$ in the parameter space of the $h_{x}-h_{z}$ plane.

The geometric phases for the trajectory of $\left(h_{x}, h_{z}\right)$ encircling an ordinary EP2 with right chirality and encircling an EP3 with left chirality are depicted in Figs. 6 and 7, respectively. In both cases, the coalescence-associated energy levels switch after encircling the EP once and are restored to their original values after encircling the EP twice. The accumulated global geometric phase is $\pi$ for one circle of encircling the right chiral EP2, and the accumulated global geometric phase is $-3 \pi$ for encircling the left chiral EP3. The winding number is $w=\Theta /(m \pi)$, where $m$ is the number of coalesced levels. Therefore, the winding number for the ordinary EP2s (EP3s) is $w= \pm 1 / 2(w= \pm 1)$. The $+(-)$ sign is for the right (left) chirality.

\section{APPENDIX C: PHASE RIGIDITY}

In this Appendix, we show the band spectrum, the phase rigidity, and the scaling exponent as the gain and loss approaching the DP, the ordinary EP2, and the EP3, respectively. 
The phase rigidity $[85,86]$ of an energy level $r=$ $\left|\left\langle\psi_{n}^{*} \mid \psi_{n}\right\rangle /\left\langle\psi_{n} \mid \psi_{n}\right\rangle\right|$ has a scaling law in the vicinity of EPs in the form of $\left|r_{\mathrm{EP}}-r\right| \propto\left(\gamma_{\mathrm{EP}}-\gamma\right)^{\nu}$. At $\Phi=0$, the degeneracies are a two-level diabolic point at $( \pm 1,0)$ in the Hermitian lattice of $\gamma=0$. In Fig. 8, the DPs appear when $\gamma_{\mathrm{DP}}=0$; two energy levels become a complex conjugation pair, the imaginary part of which changes linearly in the vicinity of DPs. The phase rigidities for all three levels are $r_{\mathrm{DP}}=1.0$, and the scaling exponents are all identical, $v=2.0$.

In Fig. 9, the energy level, phase rigidity, and scaling law are depicted for the ordinary EP2 of $\Phi=0, J=1$ at $\left(h_{x}, h_{z}\right)=(\sqrt{2} / 4,0)$. The system has one real energy, and two energy levels coalesce at the EP2. The phase rigidities of the coalesced levels are $r_{\mathrm{EP}}=0$ at the EP2 $\gamma_{\mathrm{EP}}=\sqrt{2} / 2$, and the corresponding scaling exponent is $v=0.5$; the third level, which did not participate in the coalescence, has a scaling exponent $v=1.0$.

In Fig. 10, the energy level, the phase rigidity, and the scaling law are depicted for the EP3 of $J=1, \Phi=\pi / 2$ at $\left(h_{x}, h_{z}\right)=(\sqrt{2} / 2,0)$. The system has chiral symmetry; the energy levels are symmetric about zero and exhibit a squareroot dependence on the non-Hermiticity as $\pm \sqrt{2-\gamma^{2}}$. The phase rigidities of three eigenstates equal $r_{\mathrm{EP}}=\sqrt{2} / 2$ at the EP3 $\gamma_{\mathrm{EP}}=\sqrt{2}$. The scaling exponents are $v=1$.
[1] T. Kato, Perturbation Theory of Linear Operators, Springer classics in mathematics (Springer, New York, 1980).

[2] C. M. Bender, Rep. Prog. Phys. 70, 947 (2007).

[3] N. Moiseyev, Non-Hermitian Quantum Mechanics (Cambridge University Press, Cambridge, 2011).

[4] M.-A. Miri and A. Alù, Science 363, eaar7709 (2019).

[5] M. V. Berry, Czech. J. Phys. 54, 1039 (2004).

[6] S. Klaiman, U. Günther, and N. Moiseyev, Phys. Rev. Lett. 101, 080402 (2008).

[7] K. G. Makris, R. El-Ganainy, D. N. Christodoulides, and Z. H. Musslimani, Phys. Rev. Lett. 100, 103904 (2008).

[8] A. Guo, G. J. Salamo, D. Duchesne, R. Morandotti, M. VolatierRavat, V. Aimez, G. A. Siviloglou, and D. N. Christodoulides, Phys. Rev. Lett. 103, 093902 (2009); C. E. Rüter, K. G. Makris, R. El-Ganainy, D. N. Christodoulides, M. Segev, and D. Kip, Nat. Phys. 6, 192 (2010).

[9] B. Peng, S. K. Özdemir, F. Lei, F. Monifi, M. Gianfreda, G. L. Long, S. Fan, F. Nori, C. M. Bender, and L. Yang, Nat. Phys. 10, 394 (2014).

[10] L. Chang, X. Jiang, S. Hua, C. Yang, J. Wen, L. Jiang, G. Li, G. Wang, and M. Xiao, Nat. Photon. 8, 524 (2014).

[11] Y. D. Chong, L. Ge, H. Cao, and A. D. Stone, Phys. Rev. Lett. 105, 053901 (2010); W. Wan, Y. Chong, L. Ge, H. Noh, A. D. Stone, and H. Cao, Science 331, 889 (2011); Y. Sun, W. Tan, H.-Q. Li, J. Li, and H. Chen, Phys. Rev. Lett. 112, 143903 (2014)

[12] A. Regensburger, C. Bersch, M.-A. Miri, G. Onishchukov, D. N. Christodoulides, and U. Peschel, Nature (London) 488, 167 (2012).

[13] L. Feng, Y.-L. Xu, W. S. Fegadolli, M.-H. Lu, J. E. B. Oliveira, V. R. Almeida, Y.-F. Chen, and A. Scherer, Nat. Mater. 12, 108 (2013)

[14] R. Fleury, D. Sounas, and A. Alù, Nat. Commun. 6, 5905 (2015)

[15] L. Xiao, X. Zhan, Z. H. Bian, K. K. Wang, X. Zhang, X. P. Wang, J. Li, K. Mochizuki, D. Kim, N. Kawakami, W. Yi, H. Obuse, B. C. Sanders, and P. Xue, Nat. Phys. 13, 1117 (2017).

[16] S. Longhi, Europhys. Lett. 120, 64001 (2017).

[17] L. Feng, R. El-Ganainy, and L. Ge, Nat. Photon. 11, 752 (2017).

[18] R. El-Ganainy, K. G. Makris, M. Khajavikhan, Z. H. Musslimani, S. Rotter, and D. N. Christodoulides, Nat. Phys. 14, 11 (2018).

[19] S. K. Özdemir, S. Rotter, F. Nori, and L. Yang, Nat. Mater. 18, 783 (2019).
[20] S. K. Gupta, Y. Zou, X.-Y. Zhu, M.-H. Lu, L.-J. Zhang, X.-P. Liu, and Y.-F. Chen, Adv. Mater., 1903639 (2019).

[21] J. Wiersig, Phys. Rev. Lett. 112, 203901 (2014).

[22] M. Am-Shallem, R. Kosloff, and N. Moiseyev, New J. Phys. 17, 113036 (2015).

[23] Z. P. Liu, J. Zhang, S. K. Özdemir, B. Peng, H. Jing, X. Y. Lü, C. W. Li, L. Yang, F. Nori, and Y. X. Liu, Phys. Rev. Lett. 117, 110802 (2016).

[24] W. Chen, S. K. Özdemir, G. Zhao, J. Wiersig, and L. Yang, Nature (London) 548, 192 (2017).

[25] H. Hodaei, A. U. Hassan, S. Wittek, H. Garcia-Gracia, R. El-Ganainy, D. N. Christodoulides, and M. Khajavikhan, Nature (London) 548, 187 (2017).

[26] A. A. Mailybaev, O. N. Kirillov, and A. P. Seyranian, Phys. Rev. A 72, 014104 (2005).

[27] C. Dembowski, B. Dietz, H.-D. Gräf, H. L. Harney, A. Heine, W. D. Heiss, and A. Richter, Phys. Rev. Lett. 90, 034101 (2003).

[28] C. Dembowski, B. Dietz, H.-D. Gräf, H. L. Harney, A. Heine, W. D. Heiss, and A. Richter, Phys. Rev. E 69, 056216 (2004).

[29] R. Uzdin, A. Mailybaev, and N. Moiseyev, J. Phys. A 44, 435302 (2011).

[30] M. V. Berry and R. Uzdin, J. Phys. A 44, 435303 (2011).

[31] W. D. Heiss, J. Phys. A 45, 444016 (2012).

[32] S.-Y. Lee, J.-W. Ryu, S. W. Kim, and Y. Chung, Phys. Rev. A 85, 064103 (2012).

[33] T. J. Milburn, J. Doppler, C. A. Holmes, S. Portolan, S. Rotter, and P. Rabl, Phys. Rev. A 92, 052124 (2015).

[34] T. E. Lee, Phys. Rev. Lett. 116, 133903 (2016).

[35] K. Ding, G. Ma, M. Xiao, Z. Q. Zhang, and C. T. Chan, Phys. Rev. X 6, 021007 (2016).

[36] L. Jin, Phys. Rev. A 97, 012121 (2018).

[37] B. Zhen, C. W. Hsu, Y. Igarashi, L. Lu, I. Kaminer, A. Pick, S.-L. Chua, J. D. Joannopoulos, and M. Soljačić, Nature (London) 525, 354 (2015).

[38] J. Doppler, A. A. Mailybaev, J. Böhm, U. Kuhl, A. Girschik, F. Libisch, T. J. Milburn, P. Rabl, N. Moiseyev, and S. Rotter, Nature (London) 537, 76 (2016).

[39] H. Xu, D. Mason, L. Jiang, and J. G. E. Harris, Nature (London) 537, 80 (2016).

[40] A. U. Hassan, B. Zhen, M. Soljačić, M. Khajavikhan, and D. N. Christodoulides, Phys. Rev. Lett. 118, 093002 (2017).

[41] X.-L. Zhang, S. Wang, B. Hou, and C. T. Chan, Phys. Rev. X 8, 021066 (2018). 
[42] Q. Zhong, M. Khajavikhan, D. N. Christodoulides, and R. ElGanainy, Nat. Commun. 9, 4808 (2018).

[43] W. D. Heiss, J. Phys. A 41, 244010 (2008).

[44] E. M. Graefe, U. Gnther, H. J. Korsch, and A. E. Niederle, J. Phys. A 41, 255206 (2008).

[45] X. Z. Zhang, L. Jin, and Z. Song, Phys. Rev. A 85, 012106 (2012).

[46] Q. Zhong, D. N. Christodoulides, M. Khajavikhan, K. G. Makris, and R. El-Ganainy, Phys. Rev. A 97, 020105(R) (2018).

[47] G. Demange and E.-M. Graefe, J. Phys. A 45, 025303 (2012).

[48] G. Montambaux, F. Piéchon, J.-N. Fuchs, and M. O. Goerbig, Phys. Rev. B 80, 153412 (2009); L.-K. Lim, J.-N. Fuchs, and G. Montambaux, Phys. Rev. Lett. 108, 175303 (2012).

[49] L. Tarruell, D. Greif, T. Uehlinger, G. Jotzu, and T. Esslinger, Nature (London) 483, 302 (2012).

[50] J.-M. Hou, Phys. Rev. B 89, 235405 (2014).

[51] S. Lin, L. Jin, and Z. Song, Phys. Rev. B 99, 165148 (2019).

[52] H. Shen, B. Zhen, and L. Fu, Phys. Rev. Lett. 120, 146402 (2018).

[53] X.-L. Zhang and C. T. Chan, Phys. Rev. A 98, 033810 (2018).

[54] D. Leykam, K. Y. Bliokh, C. Huang, Y. D. Chong, and F. Nori, Phys. Rev. Lett. 118, 040401 (2017).

[55] L. Jin and Z. Song, Phys. Rev. Lett. 121, 073901 (2018).

[56] M. Hafezi, E. A. Demler, M. D. Lukin, and J. M. Taylor, Nat. Phys. 7, 907 (2011).

[57] K. Fang, Z. Yu, and S. Fan, Phys. Rev. Lett. 108, 153901 (2012).

[58] M. Hafezi, S. Mittal, J. Fan, A. Migdall, and J. M. Taylor, Nat. Photonics 7, 1001 (2013).

[59] E. Li, B. J. Eggleton, K. Fang, and S. Fan, Nat. Commun. 5, 3225 (2014).

[60] L. Lu, J. D. Joannopoulos, and M. Soljačić, Nat. Photon. 8, 821 (2014).

[61] N. Goldman, J. C. Budich, and P. Zoller, Nat. Phys. 12, 639 (2016).

[62] N. R. Cooper, J. Dalibard, and I. B. Spielman, Rev. Mod. Phys. 91, 015005 (2019).

[63] T. Ozawa, H. M. Price, A. Amo, N. Goldman, M. Hafezi, L. Lu, M. C. Rechtsman, D. Schuster, J. Simon, O. Zilberberg, and I. Carusotto, Rev. Mod. Phys. 91, 015006 (2019).

[64] C. Weeks and M. Franz, Phys. Rev. B 82, 085310 (2010).

[65] S. Mittal, J. Fan, S. Faez, A. Migdall, J. M. Taylor, and M. Hafezi, Phys. Rev. Lett. 113, 087403 (2014).

[66] S. Mittal, S. Ganeshan, J. Fan, A. Vaezi, and M. Hafezi, Nat. Photon. 10, 180 (2016).

[67] C. Poli, M. Bellec, U. Kuhl, F. Mortessagne, and H. Schomerus, Nat. Commun. 6, 6710 (2015).

[68] S. Weimann, M. Kremer, Y. Plotnik, Y. Lumer, S. Nolte, K. G. Makris, M. Segev, M. C. Rechtsman, and A. Szameit, Nat. Mater. 16, 433 (2017).

[69] A. Cerjan, S. Huang, M. Wang, K. P. Chen, Y. Chong, and M. C. Rechtsman, Nat. Photon. 13, 623 (2019).
[70] H. Ramezani, Phys. Rev. A 96, 011802(R) (2017).

[71] D. Leykam, S. Flach, and Y. D. Chong, Phys. Rev. B 96, 064305 (2017).

[72] L. Jin, Phys. Rev. A 99, 033810 (2019); S. M. Zhang and L. Jin, ibid. 100, 043808 (2019).

[73] The AB phase $\Phi$ has a period of $2 \pi$. In the discussion, we have taken $\Phi(\bmod 2 \pi)$ and consider $\Phi \in[-\pi, \pi]$.

[74] H. Huang, K.-H. Jin, and F. Liu, Phys. Rev. B 98, 121110(R) (2018).

[75] H. Liu, J.-T. Sun, C. Cheng, F. Liu, and S. Meng, Phys. Rev. Lett. 120, 237403 (2018).

[76] M. Milićević, G. Montambaux, T. Ozawa, O. Jamadi, B. Real, I. Sagnes, A. Lemaître, L. Le Gratiet, A. Harouri, J. Bloch, and A. Amo, Phys. Rev. X 9, 031010 (2019).

[77] J. C. Garrison and E. M. Wright, Phys. Lett. A 128, 177 (1988).

[78] M. V. Berry, Ann. N.Y. Acad. Sci. 755, 303 (1995).

[79] A. Mead, Rev. Mod. Phys. 64, 51 (1984).

[80] M. S. Sarandy and D. A. Lidar, Phys. Rev. A 73, 062101 (2006).

[81] R. Yu, X. L. Qi, A. Bernevig, Z. Fang, and X. Dai, Phys. Rev. B 84, 075119 (2011).

[82] A. A. Soluyanov and D. Vanderbilt, Phys. Rev. B 85, 115415 (2012).

[83] A. A. Soluyanov and D. Vanderbilt, Phys. Rev. B 83, 035108 (2011).

[84] S.-D. Liang and G.-Y. Huang, Phys. Rev. A 87, 012118 (2013).

[85] H. Eleuch and I. Rotter, Eur. Phys. J. D 69, 230 (2015); 69, 229 (2015); Phys. Rev. A 93, 042116 (2016).

[86] I. Rotter, J. Phys. A 42, 153001 (2009); arXiv:1707.03298; I. Rotter and J. P. Bird, Rep. Prog. Phys. 78, 114001 (2015).

[87] H. Zhou, C. Peng, Y. Yoon, C. W. Hsu, K. A. Nelson, L. Fu, J. D. Joannopoulos, M. Soljačić, and B. Zhen, Science 359, 1009 (2018).

[88] X. Huang, Y. Lai, Z. H. Hang, H. Zheng, and C. T. Chan, Nat. Mater. 10, 582 (2011).

[89] S. Lin and Z. Song, Phys. Rev. A 96, 052121 (2017).

[90] N. P. Armitage, E. J. Mele, and A. Vishwanath, Rev. Mod. Phys. 90, 015001 (2018).

[91] D. Guzmán-Silva, C. Mejía-Cortés, M. A. Bandres, M. C. Rechtsman, S. Weimann, S. Nolte, M. Segev, A. Szameit, and R. A. Vicencio, New J. Phys. 16, 063061 (2014).

[92] Y.-X. Xiao, Z.-Q. Zhang, Z. H. Hang, and C. T. Chan, Phys. Rev. B 99, 241403(R) (2019).

[93] L. Jin and Z. Song, Phys. Rev. B 99, 081103(R) (2019); H. C. Wu, L. Jin, and Z. Song, ibid. 100, 155117 (2019).

[94] X. Z. Zhang and Z. Song, Ann. Phys. (NY) 339, 109 (2013); C. Li, L. Jin, and Z. Song, Phys. Rev. A 95, 022125 (2017).

[95] L. Jin, Phys. Rev. A 96, 032103 (2017). 